\title{
INDOOR LOCATION CHALLENGES. AN AUGMENTED REALITY APPROACH
}

\author{
Alexandru Marius OBRETIN \\ Bucharest University of Economic Studies, Romania \\ alexandru.obretin93@gmail.com
}

\begin{abstract}
Romania's capital city is one of the most endangered cities in Europe due to its proximity to the Vrancea seismic region. Over the years both authorities and researchers have tried to understand the root causes, implement monitoring systems and mitigate risks. So far, the efforts were concentrated on estimations and pre-disaster solutions that can limit the impact. In contrast, modern technologies provide stakeholders the opportunity to develop relevant post-disaster decision support systems that facilitate reaction after an incident. Indoor location systems have multiple applications in emergency mitigation context, the current paper detailing the building evacuation scenario. A software solution that relies on smartphone capabilities to determine the shortest evacuation route is further introduced. The application provides convenient workarounds for known technological limitations, grants high accuracy and avoids the costs of traditional sensors-based indoor location systems. Nevertheless, this solution is tailored to the user, instead of the rescue teams, which to the best of our knowledge is the first academic initiative of its kind in Romania.
\end{abstract}

Keywords: Augmented Reality, Building Evacuation, Disasters, Indoor Positioning System JEL classification: $\mathrm{O} 320, \mathrm{O} 350$

DOI: $10.12948 / \mathrm{ie} 2019.06 .06$

\section{Introduction}

Bucharest, the capital city of Romania is the $10^{\text {th }}$ largest urban area in Europe, as well as one of the most important economic and social spots in the Eastern side of the continent. Being home for 2.106144 million inhabitants $(9.469 \%$ of Romania's total population) as of January $1^{\text {st }}, 2016$, Bucharest and its metropolitan area produced more than $25 \%$ of Romania's GDP in 2017, according to the National Commission for Strategy and Prognosis.

Besides, the Romania's capital is also one of the most endangered major European cities when comes to natural disasters. Located closely to the Vrancea intermediate-depth seismic source region, Bucharest confronts statistically with 2 or 3 major seismic activities per century.

The most devastating Vrancea earthquakes in recent history killed more than 1500 people in Bucharest which influenced over the years the urban development strategies and determined an increased interest in the subject from both the local authorities and the academic environment. Various studies have analysed the geomorphological particularities, categorized the existing building stock and involved Romania in international seismic research teams. Technical advancement led to more powerful processing units, boosted the development of complex deep learning algorithms able to handle large amounts of information and maturated mixed reality technologies, which combined can provide a better understanding of the risks. Worldwide, modern technologies like virtual reality (VR) and augmented reality (AR) proved useful in emergency mitigation scenarios and have been integrated in educational initiatives that aimed to train people in complex scenarios like fires, floods or terrorist attacks. The 
researchers demonstrated that brain cells get tricked and cannot distinguish between a VR and a real-life scenario, such experiments enhancing learning [1].

\section{Literature review}

As no one can question a future major disaster, the Romanian researchers assisted local institutions to facilitate mitigation measures and followed several directions regarding the consequences of a moment magnitude $\mathrm{Mw} \geq 7.5$ earthquake for Bucharest.

On the one hand, a joint academic research, including specialists from both the National Institute for Earth Physics (NIEP) and the Technical University of Civil Engineering, tried to comprehensively explain the particularities of Vrancea seismic region.

First, using the latest recorded information about the Romanian ground motion, the researchers developed several prediction equations for the target region, which were further involved in a deterministic spatial correlation model [2]. Second, certain seismic scenarios have considered a function of magnitude, distance from source to site and focal depth and run 2000 simulations for the city of Bucharest. The experiment determined the mean values for the economic losses between 5 and 13 billion euros. Furthermore, the time required for restoring $95 \%$ of the initial housing capacity varies from 550 to 2000 days for different intensity earthquakes [3].

On the other hand, the researchers from the University of Bucharest and the Bucharest University of Economic Studies approached the social and economic features of the inhabitants to provide a detailed representation of city's vulnerable hotspots. Their analysis revealed that undeveloped infrastructure and lack of quality education enhance the potential risk of limited disaster resilience for the inhabitants of such areas. These factors lead to social segregation, cultural, professional and demographic barriers, the study concludes [4].

Another research addressing earthquake vulnerability proved that the aged, unmaintained building stock partly overlaps the distribution pattern of social vulnerability among Bucharest citizens [5]. Consequently, the city is confronting with neighbourhoods where the residents have limited financial resources and seldom invest in consolidations.

Local authorities have recently implemented strategies and frameworks to mitigate the impact of a major disaster. Several relevant examples are the BIGSEES Catalog that improved the Romanian Seismic Design Code (P100) [6], the RO-ALERT system deployed by the Department for Emergency Situations that allows broadcasting cell phones messages to warn citizens in case of emergency, and SeisDaRo, a cutting-edge NIEP project for near real-time earthquake damage estimations.

SeisDaRo is connected to the national strong motion accelerometers network (Romanian Early Warning System) and automatically triggered once a moment magnitude $\geq 4$ earthquake is happening. It integrates 3 main modules:

- ShakeMap used for rapidly portraying the distribution and severity of the earthquake;

- HAZUS used for estimating the number of victims;

- SELENA used for building collapse impact and loss estimations.

Only 35 seconds after the initial burst, the system roughly estimates the number of victims using PAGER-RO, a Romanian specific adaptation of the USGS PAGER methodology [7]. Within 3 minutes, a detailed assessment of the earthquake is finished, resulting in graphical representations, simulations, reports and analyses. A more detailed explanation of SeisDaRo is provided by Toma-Dănilă et al [8].

Nevertheless, effective building evacuation systems, aerial scanners that can identify victims or virtual reality training rooms are at least as important as earthquake simulators and impact analysis. For example, in fires the most common cause of death is toxic smoke inhalation, rather than the burns, so in such context in-time evacuation might mean salvation. 
Several studies revealed that people exposed to emergency conditions spend more time finding a way out compared to the control sample [9]. Therefore, training corroborated with decision support systems can diminish the psychological impact the danger is inducing.

Novel decision support systems designed for building evacuation scenarios use data mining and IoT technologies, a comprehensive literature review being provided by Sattarian et al [10]. Moreover, the technological advance favoured the integration of effective deep learning algorithms with augmented reality hardware into systems capable of processing large amounts of environmental data in real-time. Indoor navigation solutions using augmented reality have been proved efficient in accurately estimating target locations. Some solutions first gather natural markers, pass them as input to computer vision algorithms that try to determine the position relative to some preliminary stored data and second involve AR for displaying details about current location and guiding the user [11]. Others use AR devices to capture images that are converted into building information models (BIM) and compared with reference representations using trained convolutional neural networks $(\mathrm{CNN})$ to identify the pipes inside the walls for facility management purposes [12]. The later kind provided high matching accuracy for image-based localization, the neurons being pretrained on large image data sets which enhances the capability to outline and learn the most relevant features of an image [13] [14]. It is worth mentioning that augmented reality has become more and more appropriate for civil engineering and environmental planning, a mobile application for flood visualisation being just an example [15], great potential being expected in education and learning.

\section{Outdoor and indoor location}

Regarding emergency situations, we have considered two main analysis perspectives.

The first is focusing on observation and prevention and takes place before an event is happening. This approach helps local authorities understand the risks, design evacuation plans and effectively allocate funds for infrastructure development.

The second is reaction-oriented and centred on what everyone exposed to an emergency might do once such situation occurs. The latter category might include efficient localization systems, up and running post disaster communication technologies or software applications that help victims leave endangered areas. For the rest of this paper we will focus on the latter category. The Global Positioning System (GPS) consists of a network of over 30 satellites that reside in Earth's orbit and can accurately offer geolocation. To provide this service, the GPS requires a target that is simultaneously in the detection range of multiple satellites. Target identification implies signal transmission from the satellite and reception from the receiver. The signal is travelling with constant speed and the time delay between transmission and reception moments is independent on satellites speed, being proportional to the distance between the satellite and the target. The exact location is further determined by a GPS receiver that aggregates the data from at least 4 satellites for accuracy. Using geolocation (Figure 1), the shortest path between two points can be calculated, while receiving real-time updates. 


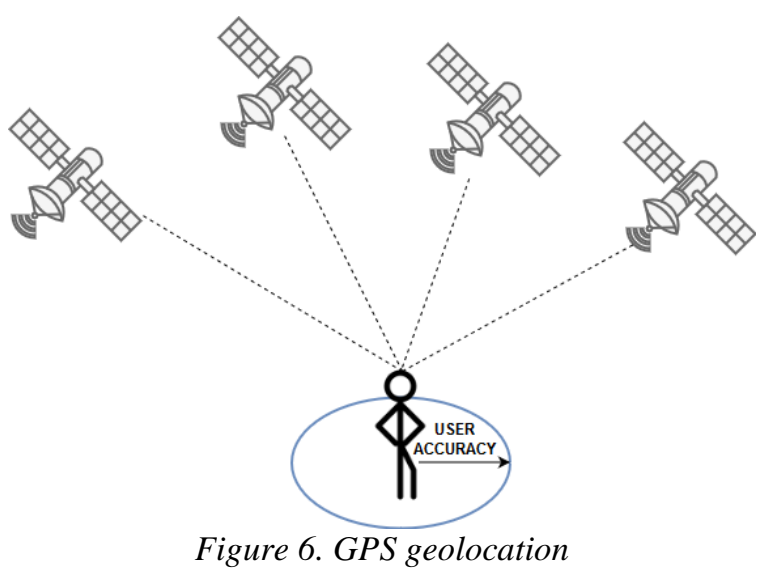

However, GPS alone becomes inefficient for indoor location purposes, being negatively influenced by underground usage, reflected microwaves by buildings or walls or signal blockage due to vegetation, constructions or chemical composition of building materials.

A solution for this issue is represented by an Indoor Positioning System (IPS), which instead of transmitting satellite microwaves, it engages wireless technologies and series of receivers that calculate the intensity of signals that cross the covered areas. Bluetooth based solutions are used for creating virtual perimeters (geo-fences) and notice proximity, while grid networks of sensors are integrated when exact location is desired. The algorithms behind often involve triangulation with known sensor positions or complex models for precise estimations that exclude statistical noise (Kalman filtering or Bayesian statistical analysis).

As sensor grids are expensive to install and estimations tend to be influenced by environmental changes, we would like to propose a different approach, convenient for building evacuation scenarios like lost individuals, fires, closed access routes, terrorist attacks or earthquakes. This approach does not offer a generic solution, since some components should be manually provided and stored in a predefined format, but it can be extrapolated if convenient.

\subsection{Indoor location challenges}

Differently from outdoor positioning systems, indoor location requires a more detailed analysis about a building. Overall, there are two main approaches for indoor positioning. One requires installation of hardware inside the building and the localization process relies on sensors and beans that acquire data when the target is in proximity. In this case, the constraints come from physical characteristics of the building, wave distribution patterns, types of building materials, level of refraction or degree of congestion. Another approach delegates the data acquisition phase to the target, which is aware only of the surroundings and the localization process is responsible for matching a restricted context to a broader landscape.

The first approach is relying on what literature calls artificial markers and it must be mentioned that the building owner is the one responsible for installing such hardware which is neither cheap, nor easy to do, considering that this network might interfere with existing ones.

The other solution removes the additional costs of sensors acquisition, but faces new challenges as target localization must be done relative to some continually updated information. Although, precise building representations are crucial, they must remain in accordance with reality to correctly assure geo-location. As a result, whenever a new change occurs, the reference representation should incorporate it. Otherwise, if a room changes its purpose, or the walls get repainted, or new markers are added, the algorithm that calculates the actual position might fail due to wrong model assumptions. 
Moreover, while the former choice is strongly related to physical radio communication principles between devices, the latter depends on smart and effective software solutions that compose an intermediate layer between the target and the information system of the building. Several studies have proved that an image captured inside an indoor environment is different from a $3 \mathrm{D}$ rendered model in terms of visual characteristics, which makes a cross-domain comparison between them more difficult. [16]

A possible solution for this issue relies on complex neural network algorithms that receive both the building information model and the actual captured image and compare layer 4 pooling weights. Such networks require a deep understanding of image processing and the neurons have to be previously trained with sample data sets.

Other challenges, according to the following study [17], are represented by the high power consumption such networks might need, the scalability - how well the process can handle large number of requests, the robustness against threats and the general applicability.

\subsection{Proposed software solution}

The informatic solution we propose comprises 4 different modules that interact with each other in different execution phases. Mainly, it relies on several public APIs, a neural network solution for accurate altitude estimations, the smartphone's front camera for information gathering and camera's application for augmented reality display. When launched, the application determines the user location, collects information and calculates the shortest evacuation route (SER) in regard with previously cloud stored data. While phone's camera continuously acquires data, the display is guiding the target to the nearest exit.

In the first development phase, the goal is to create a 2D representation of each floor for the Cybernetics, Statistics and Economic Informatics (CSIE) building in Bucharest. For this purpose, the official floor plans of the building, containing relevant information about access points, emergency exists, staircases, elevators, rooms, distances and shapes, will be uploaded in Google Indoor API and integrated in Google Maps Platform. Similar initiatives for Bucharest have been implemented so far by Promenada and MegaMall shopping centres. The API allows users from both desktop and mobile platforms to inspect the building floor plans from a side navigator, once they zoom in. (Figure 2) At the end of this phase the data will be stored in cloud and accessible for development purposes using the Google Maps API.
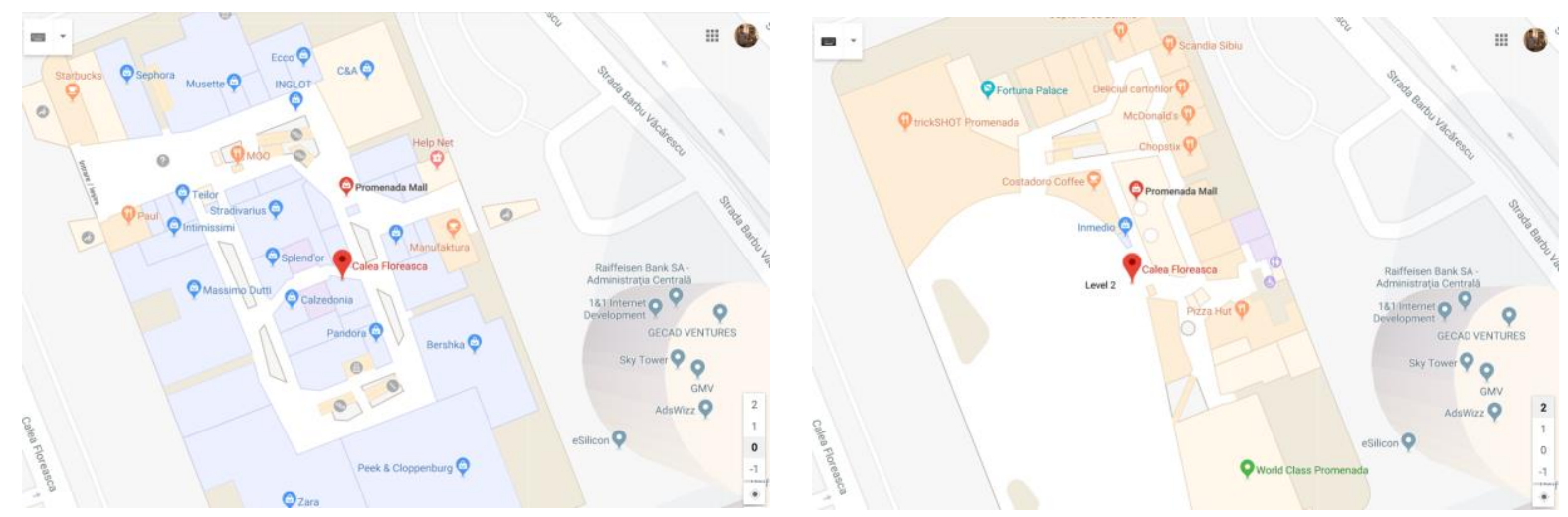

Figure 7. Google Maps floor plan for Promenada shopping center.

(left) ground floor plan. (right) second floor plan.

Next, the purpose is to accurately determine the position and the altitude in indoor context. Once someone enters the CSIE building, the informatic solution should be able to estimate the floor level and the concrete location using mobile phone capabilities. Common IPS approaches for this issue might require beacons or sensor installation. 
Although, online indoor tours are quite common nowadays, several examples being George Enescu Bucharest Philharmonic, Unirea National College in Focșani or Traian Vuia International Airport in Timișoara, they are error-prone in building evacuation scenarios.

Many constructions have symmetric floor plans, each story being a replica of the others, even in terms of construction materials and colours, this being the case also for some stories inside the CSIE building. This means that lack of numbering plates or special particularities can confuse a matching pattern algorithm that compares cloud stored information about the interior of the building with real-time images captured with the camera. Moreover, if the interior design changes while the online information remains outdated, the algorithm would fail in finding similarities. To address this issue, our solution will involve a dual approach.

First, it will invoke a service developed by Falcon and Schulzrinne [18] that trains a neural network to find out when someone enters or exits a building, based on GPS signal changes. Further, using phone's barometer, the service can determine the exact floor level.

Second, we would create an indoor tour for the CSIE building, based on a sequence of pictures. These will be correlated with the floor plans stored in Google Maps and used for computing the shortest evacuation route. Indoor tour images will be stored using Google Indoor API and will be available from Google Maps, as well.

Last, but not least, once someone launches the application to find the shortest way out from a building, it would access the phone's front camera and start gathering information about the surroundings. The neural network service would provide the exact floor level, while the camera collected data would be automatically compared with information stored on Google Maps to determine the precise coordinates. In recent years, complex surface reconstruction algorithms that enhance structure recognition have been developed and applied in VR applications [19]. Once a match is found, an augmented reality image labelling system would display arrows on the screen, guiding the user to the nearest exit. Similar purposes have already been addressed using the OpenGL library [20]. The architectural schema is detailed in Figure 3.

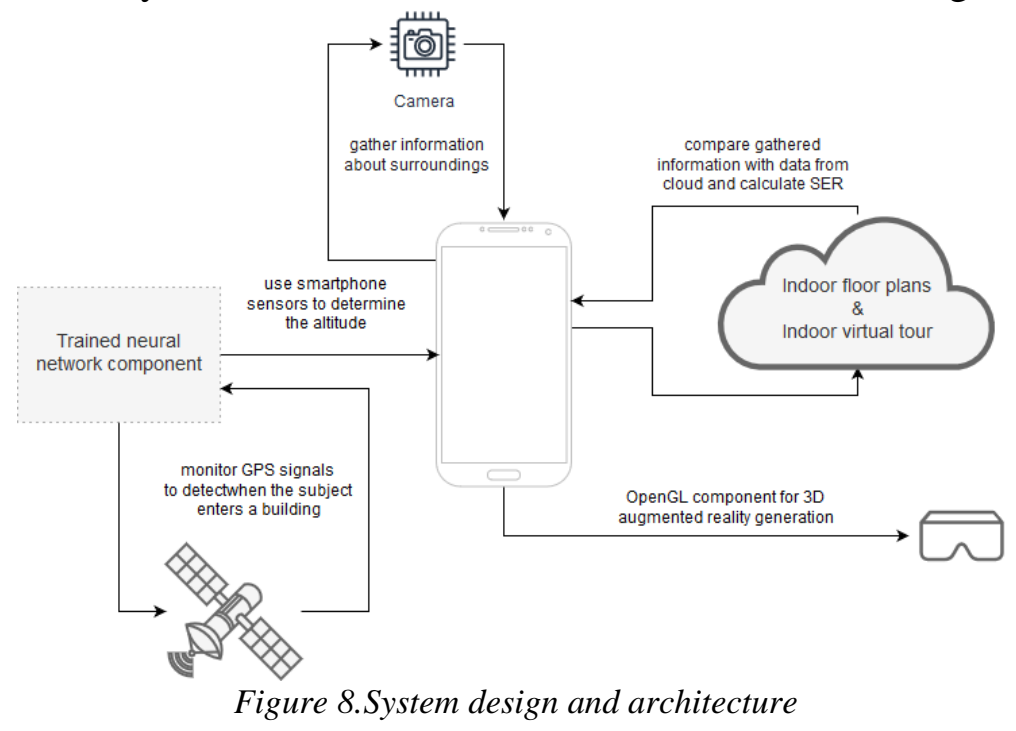

\section{Results and discussions}

To the best of our knowledge, the software solution proposed in the previous chapter is the first academic initiative of its kind in Bucharest. Other emergency mitigation examples insist on impact assessment and vulnerability exposure. Although, several components rely on acknowledged technologies and techniques, the presented components integration can be easily extended to the most important areas of public interest - universities, hospitals, public institutions, crowded shopping centres. 
Enhancements that can be considered for the proposed model refer to adding two extra modules, one that behaves like a thermometer for identifying possible fire outbreaks and a user interactive mode for scenarios that require human input, like closed doors, blocked paths or desired destination.

The status of this solution is still in the analysis and development phase, as the original architectural plans are recreated and updated. Once the correct definition of the building image models will be done, the second phase will involve publishing to Google process, followed by a technical debate about possible strengths and weaknesses of the application. We aim to identify the most important risks that have to be considered and other technical and architectural details about the reference building that might have been missed.

The development, including the implementation of debate conclusions, will be the first trial of creating a product to offer an efficient way to locate the nearest exit inside an indoor context.

\section{Conclusions}

The main purpose of this study was to perform a comprehensive literature review about emergency mitigation practices and identify the VR/AR state-of-the-art software solutions and methodologies used in this field, particularly in Romania.

The research has emphasized strong interest from the academic community towards seismology, economic impact analysis and disaster simulations due to the overall effect such natural phenomena have on local economy. As opposed, far less resources have tackled a post disaster scenario. The hereby paper focused more on the emergency evacuation strategies and delimited itself from the post-earthquake scenarios. The proposed software solution aimed to be a viable proof of concept for less complex scenarios. We understand that post-earthquake evacuation applications should consider more uncertainty and variables, while the way the application is currently designed makes it inappropriate for such scenarios.

For the moment, the architecture bypasses expensive infrastructure acquisition, avoids GPS limitations, uses mobile phone embedded technologies, provides high accuracy in terms of indoor location and empowers the user, which in emergency situations might represent a lifesaving advantage.

This paper was co-financed by The Bucharest University of Economic Studies during the $\mathrm{PhD}$ program.

\section{References}

[1] D. Lorenz, W. Armbruster, C. Vogelgesang, H. Hoffmann and A. Pattar, A new age of mass casuality education? : The InSitu project: realistic training in virtual reality environments, Der Anaesthesist, 2016.

[2] R. Văcăreanu, M. Radulian and M. Iancovici, Fore-arc and back-arc ground motion prediction model for Vrancea intermediate depth seismic source, vol. 19, Journal of Earthquake Engineering, 2015, p. 535-562.

[3] F. Pavel and R. Văcăreanu, Scenario-based earthquake risk assessment for Bucharest, Romania, vol. 20, International Journal of Disaster Risk Reduction, 2016, pp. 138-144.

[4] I. Armaș, R. Ionescu, A. Gavriș and D. Toma-Dănilă, Identifying seismic vulnerability hotspots in Bucharest, vol. 77, Applied Geography, 2016, pp. 49-63.

[5] I. Armaș, D. Toma-Dănilă, R. Ionescu and A. Gavriș, Vulnerability to Earthquake Hazard: Bucharest Case Study, vol. 8, International Journal of Disaster Risk Science, 2017, p. 182195.

[6] F. Pavel, R. Văcăreanu, J. Douglas, M. Radulian and C. Cioflan, An Updated Probabilistic Seismic Hazard Assessment for Romania and Comparison with the Approach and 
Outcomes of the SHARE Project, vol. 173, Pure and Applied Geophysics, 2016, p. 18811905.

[7] K. Jaiswal, D. Wald and M. Hearne, Estimating Casualties for Large Earthquakes Worldwide Using an Empirical Approach, U.S. Geological Survey Open-File Report OF 2009, 2009.

[8] D. Toma-Dănilă, C. Cioflan, C. Ionescu and A. Țigănescu, The near real-time system for estimating the seismic damage in Romania (SeisDaRo). Recent upgrades and results, 16th European Conference on Earthquake Engineering, 2018.

[9] C. Lijun, L. Jing and L. Nan, A virtual reality based study of indoor fire evacuation after active or passive, vol. 90, Computers in Human Behavior, 2019, pp. 37-45.

[10] M. Sattarian, J. F. R. Rezazadeh and A. Bagheri, Indoor navigation systems based on data mining techniques in, vol. 25, Wireless Networks, 2019, pp. 1385-1402.

[11] C. Koch, M. Neges, M. König and M. Abramovici, "Natural markers for augmented reality-based indoor navigation and facility maintenance," Automation in Construction, vol. 48, pp. 18-30, 2014.

[12] F. Baek, I. Ha and H. Kim, "Augmented reality system for facility management using image-based indoor localization," Automation in Construction, vol. 99, pp. 18-26, 2019.

[13] A. Kendall, M. Grimes and R. Cipolla, "PoseNet: A Convolutional Network for RealTime 6-DOF Camera Relocalization," in IEEE International Conference on Computer Vision (ICCV), Santiago, Chile, 2015.

[14] F. Walch, C. Hazirbas and L. Leal-Taixé, "Image-based localization using LSTMs for structured feature correlation," in IEEE International Conference on Computer Vision (ICCV), Venice, Italy, 2017.

[15] P. Haynes, S. Hehl-Lange and E. Lange, "Mobile Augmented Reality for Flood Visualisation," Environmental Modelling \& Software, vol. 109, pp. 380-389, 2018.

[16] I. Ha, H. Kim, S. Park and H. Kim, "Image retrieval using BIM and features from pretrained VGG network for indoor localization," Building and Environment, vol. 140, pp. 23-31, 2018.

[17] J. De Brabanter, P. Verhoeve and J. Wyffels, "Using a decision tree for real-time distributed indoor localization in healthcare environments," in International Conference on Development and Application Systems (DAS), 2014.

[18] W. Falcon and H. Schulzrinne, Predicting floor level for 911 calls with neural networks and smart phone sensor data, International Conference on Learning Representations, 2018.

[19] M. Lhuillier, Surface reconstruction from a sparse point cloud by enforcing visibility consistency and topology constraints, vol. 175, Computer Vision and Image Understanding, 2018, pp. 52-71.

[20] J. Ahn and R. Han, An indoor augmented-reality evacuation system for the Smartphone using, Human-centric Computing and Information Sciences, 2012. 